Im „Journal Club“ werden Originalarbeiten aus der internationalen Fachliteratur referiert und kommentiert.

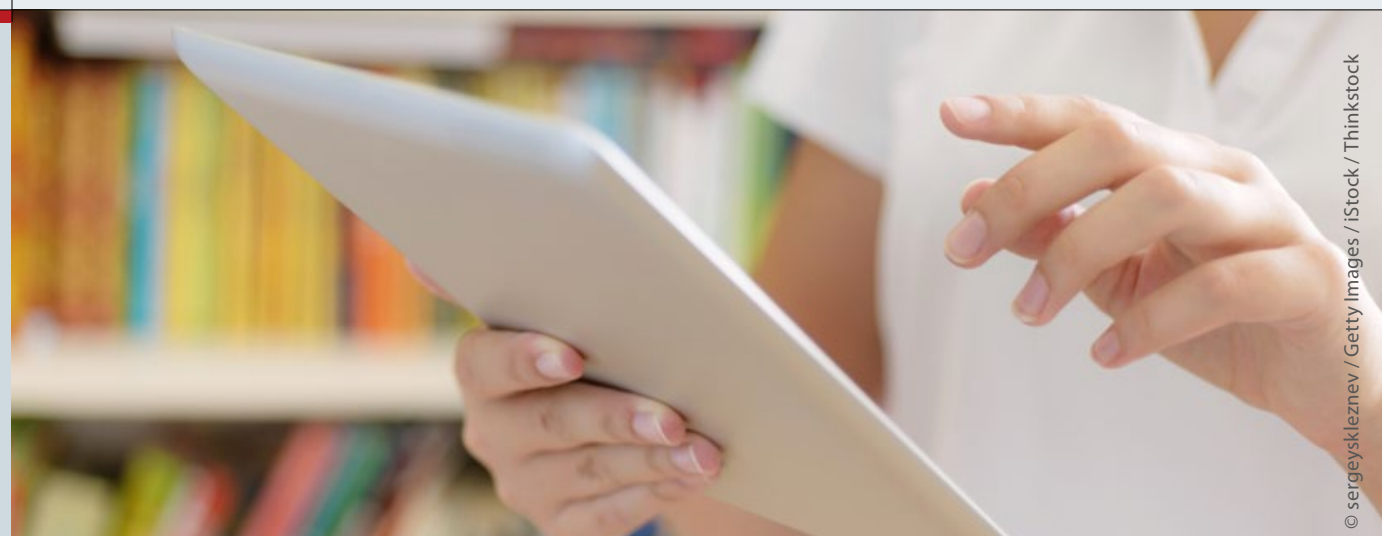

\section{Erhöhtes Sturzrisiko in Folge der Chemotherapie}

\section{Viele Jahre nach einer Chemotherapie haben Frauen noch immer Symptome einer durch die Krebsbehandlung induzierten peripheren Neuropathie. Im Vergleich zu Patientinnen ohne diese Symptome ist bei ihnen zudem das Risiko für Stürze verdoppelt.}

B isherige Untersuchungen zur Chemotherapie-induzierten peripheren Neuropathie stützten sich vor allem auf die Angaben der betroffenen Patienten. Ärzte der Oregon Health \& Science University in Portland haben deshalb in einer Studie sowohl anhand der subjektiven Angaben von Krebspatientinnen als auch anhand objektiver Messungen die Folgen der anhaltenden Neuropathie analysiert. Die Patientinnen waren Teilnehmerinnen von vier Studien und zum Zeitpunkt der Krebsdiagnose älter als 50 . Sie hatten keine Metastasen und keine neurologischen Symptome und waren mehr oder weniger Bewegungsmuffel.
An der Universität wurden sie Jahre nach der Diagnose zum einen nach ihrer körperlichen Fitness, zum anderen nach möglichen Stürzen gefragt. Außerdem wurden objektive Messungen vorgenommen, etwa der körperlichen Leistungsfähigkeit, sowie eine Ganganalyse (Geschwindigkeit und Muster).

\section{Die meisten hatten Brustkrebs}

Mehr als 500 Patientinnen im Alter von $63 \pm 6$ Jahren nahmen an der Studie teil, die meisten hatten Brustkrebs (78\%). Sie waren mit einem BMI von 29,6 $\pm 6,6 \mathrm{~kg} /$ $\mathrm{m}^{2}$ adipös. Nur $11 \%$ hatten außer Krebs auch Diabetes. Die Krebsdiagnose lag

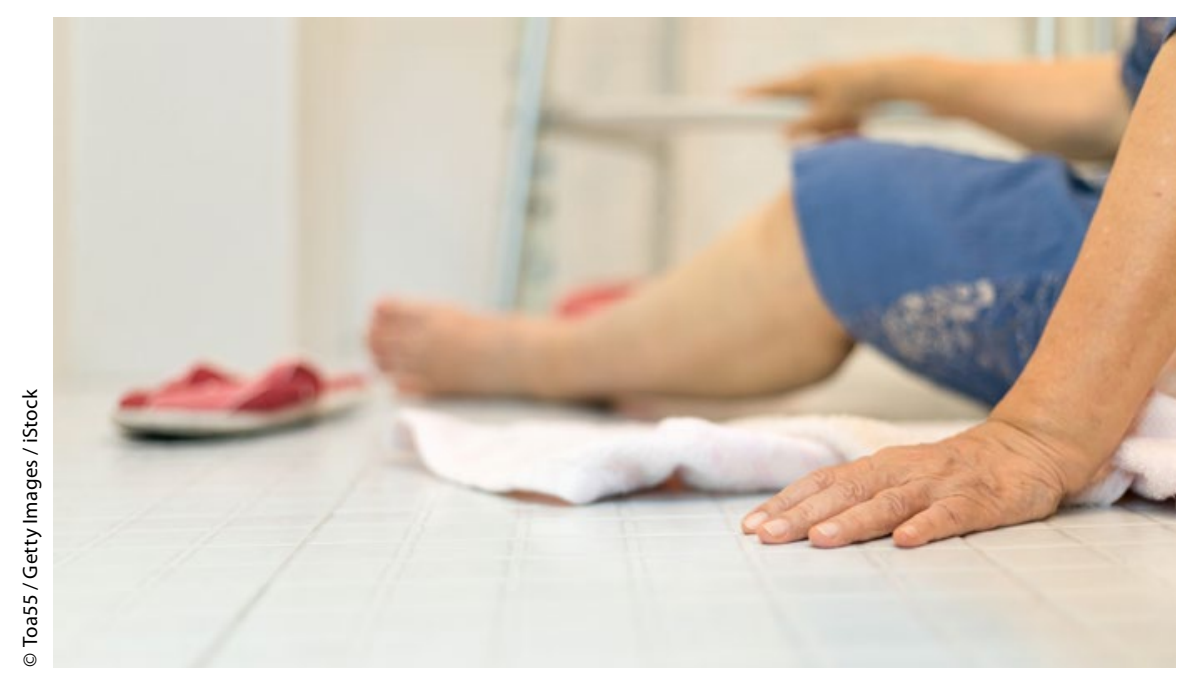

drei Monate bis 33 Jahre zurück. Nach Angaben der Ärzte berichteten durchschnittlich sechs Jahre nach der Krebstherapie $47 \%$ noch immer über einen Sensibilitätsverlust in den Beinen. Sowohl den Eigenberichten als auch den objektiven Messungen zufolge war die körperliche Fitness in der Gruppe mit peripherer Neuropathie signifikant schlechter. Ausnahme: die maximale Beinmuskelkraft und die Unterstützungsfläche des Körpers während des Gehens.

Im Vergleich zu Patientinnen ohne periphere Neuropathie gingen die Frauen mit der neurologischen Störung langsamer und in kürzeren Schritten. Sie berichteten, körperlich deutlich eingeschränkter zu sein und mussten öfter stehen bleiben, um das Gleichgewicht zu stabilisieren. Außerdem war bei ihnen die Wahrscheinlichkeit, innerhalb des vergangenen Jahres gestürzt zu sein, um fast das Zweifache höher als in der Vergleichsgruppe. Etwas geringer lag die Wahrscheinlichkeit für Stürze mit Verletzungsfolgen. Das Sturzrisiko war zudem höher, je ausgeprägter die Neuropathiesymptome waren.

Nach Angaben der US-Ärzte sprechen die Ergebnisse der Studie dafür, so früh wie möglich Chemotherapie-induzierte periphere Neuropathien zu erkennen und entsprechend zu behandeln, um das Fortschreiten zu unterbinden und damit die körperliche Funktion zu erhalten. So könnten letztlich auch Stürze verhindert werden.

(pl)

Winters-Stone KM et al. Falls, Functioning, and Disability Among Women With Persistent Symptoms of Chemotherapy-Induced Peripheral Neuropathy. JCO 2017; online 6. Juni. doi: 10.1200/JCO.2016.71.3552 